Cronfa - Swansea University Open Access Repository

This is an author produced version of a paper published in :

Nano Research

Cronfa URL for this paper:

http://cronfa.swan.ac.uk/Record/cronfa31840

\title{
Paper:
}

Li, L. \& Zhang, Y. (2017). Controlling the luminescence of monolayer MoS2 based on the piezoelectric effect. Nano Research

http://dx.doi.org/10.1007/s12274-017-1457-y

This article is brought to you by Swansea University. Any person downloading material is agreeing to abide by the terms of the repository licence. Authors are personally responsible for adhering to publisher restrictions or conditions. When uploading content they are required to comply with their publisher agreement and the SHERPA RoMEO database to judge whether or not it is copyright safe to add this version of the paper to this repository. http://www.swansea.ac.uk/iss/researchsupport/cronfa-support/ 


\section{TABLE OF CONTENTS (TOC)}

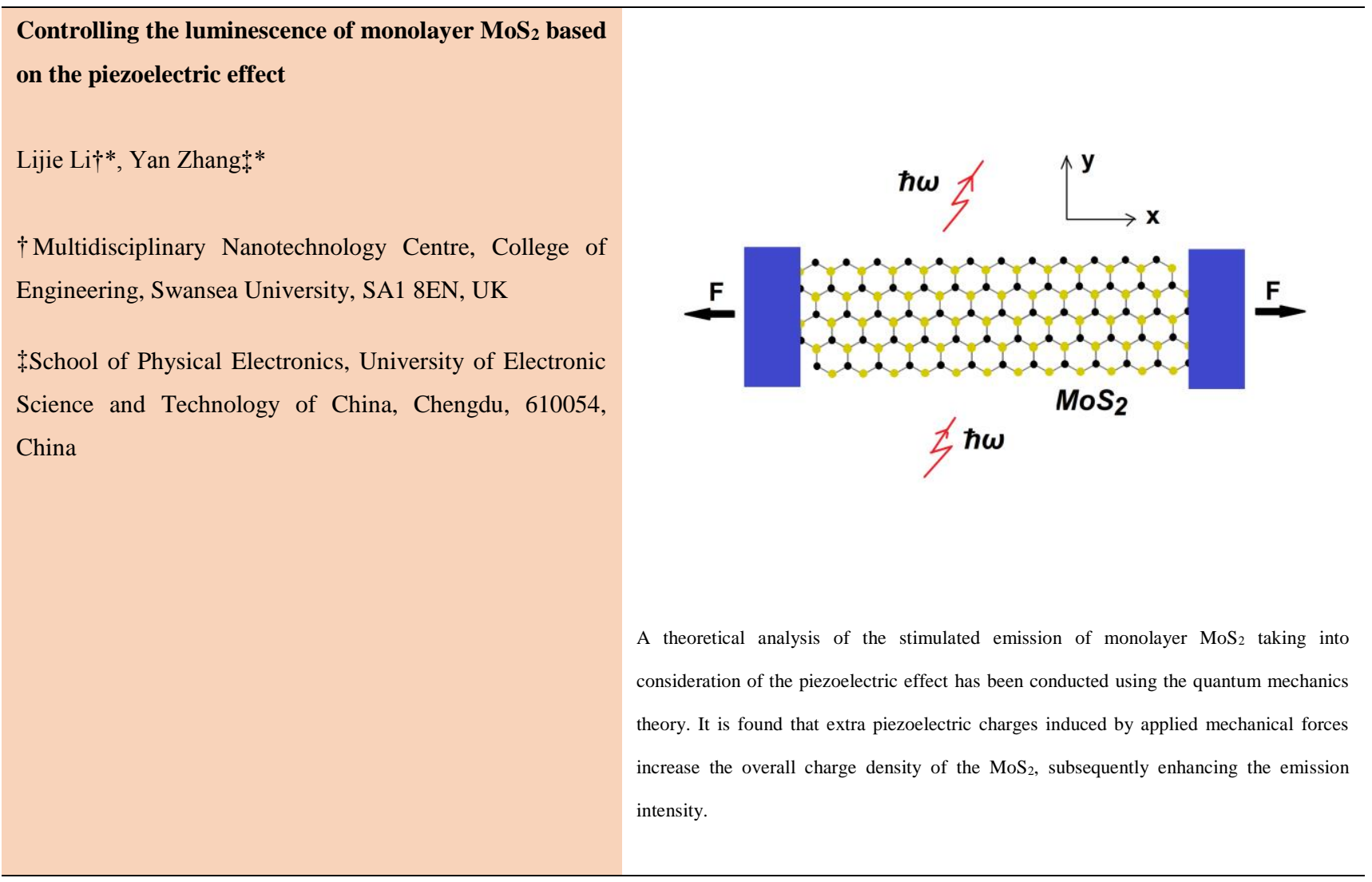




\section{Controlling the lumines cence of monolayer $\mathrm{MoS}_{2}$ bas ed on the piezoelectric effect}

Lijie $\operatorname{Li}^{1}(\square)$, and Yan Zhang ${ }^{2}(\square)$

${ }^{1}$ Multidisciplinary Nanotechnology Centre, College of Engineering, Swansea University, Bay Campus, SA1 8EN, UK

${ }^{2}$ School of Physical Electronics, University of Electronic Science and Technology of China, Chengdu, 610054, China

Received: 16 November 2016

Revised: day month year

Accepted: day month year (automatically inserted by the publisher)

(C) Tsinghua University Press and Springer-Verlag Berlin Heidelberg 2016

\section{KEYWORDS}

Piezo-phototronics

Luminescence

$\mathrm{MoS}_{2}$

\begin{abstract}
We report on manipulating the stimulated emission of the monolayer molybdenum disulfide $\left(\mathrm{MoS}_{2}\right)$ with the piezoelectric effect. The analysis is based on quantum mechanics theory. Stimulated emission of this two-dimensional material has been simulated to establish the relation between the total emission rate and the energy of the photon excitation. We demonstrate that the piezoelectric induced charges enhance the emission rate by changing the carrier concentration. It is found that the emission intensity is proportional to the carrier density in the low-density range, and it eventually reaches to a steady value in the high-density region. Externally applied mechanical force also leads to change of the second harmonic generation from the monolayer $\mathrm{MoS}_{2}$.
\end{abstract}

\section{Introduction}

The transition-metal dichalcogenide semiconductor $\mathrm{MoS}_{2}$ has caused great attention attributed to its superior electronic, optical, and catalytic properties [1] [2] [3] [4]. It has been found that the monolayer has the distinctive semiconductor property - direct bandgap as compared to its bulk form [2]. This property will significantly enhance the photon emissions against the indirect bandgap exhibited in the bulk form, which will have great potential in applications such as light emitting devices. Pioneer study on piezotronics of the $\mathrm{MoS}_{2}$ has been conducted to unveil its another important property -

Address correspondence to Lijie Li, L.Li@swansea.ac.uk; Yan Zhang, zhangyan@uestc.edu.cn

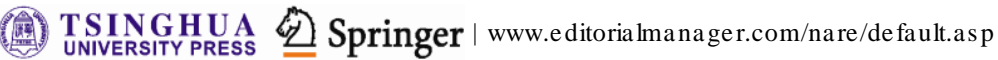


piezoelectricity [5] [6] [7], which promotes this material in energy harvesting and sensor applications. Though the research on photoluminescence of the monolayer $\mathrm{MoS}_{2}$ has been experimentally investigated in Ref. [8], theoretical research has not been conducted, especially for the non-trivial aspect of piezoelectric enhanced luminescence, which leads to the emerging field of piezo-phototronics [9] [10] [11]. Interesting approaches of controlling luminescence through combined magnetic and piezophotonic has been reported in [12]. A comprehensive review on tuning the luminescence with various methods was presented in [13]. From the theory, there are two known parameters affecting the stimulated luminescence, one is the charge density, the other is the bandgap of the material. In majority of previous reported strategies, the ultimate aim was to tune the charge density in order to control the intensity of the luminescence. In essence it is aimed to increase the amount of electrons available that transit from states of higher band to states of lower band. The bandgap of the material mainly determines emission wavelength. In this work we analyze the stimulated emission of the monolayer $\mathrm{MoS}_{2}$ based on the inelastic photon-electron interaction. Due to the direct bandgap of the monolayer $\mathrm{MoS}_{2}$, our approach of using the Fermi golden rule is taken to analyze the light emission rate. The theoretical method has been used in previous study in zinc oxide nanowires [14]. Our aim in this work is to theoretically explore the impact on the luminescence attributed to photonic and electronic parameters i.e. bandgap, incident photon energy. Furthermore, the emission rate depending on the piezoelectric effect for this material requires urgent exploration, especially since the interesting piezo-phototronic concept has been proposed recently. The advantage of using piezoelectric is its dynamical tunability of piezoelectric charges created by applied strain. For 2D materials fixed on substrate, doped 2D materials will change luminescence, for example, luminescence wavelength can be extended effectively by rare-earth Er-doped $\mathrm{MoS}_{2}$ [15]. The mechanism of the possible enhancement on the luminescence by the piezoelectric effect will be detailed in the following sections of this paper.

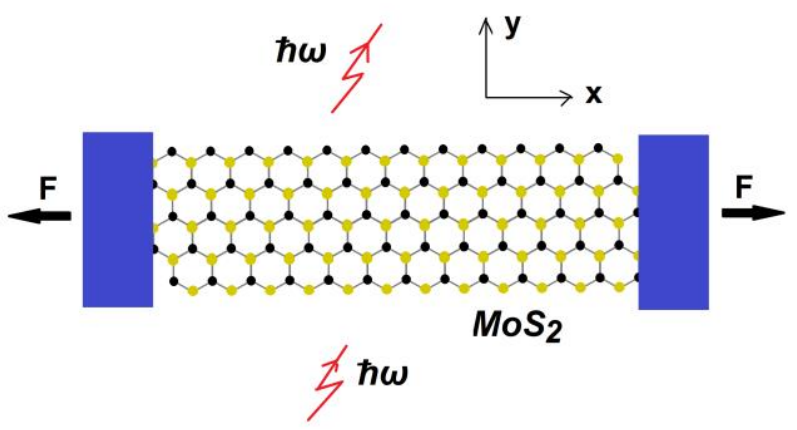

Figure 1. Schematic of the single layer $\mathrm{MoS}_{2}$ stretched by the force $F$, affecting the stimulated emission.

\section{Theory}

As a beam of light with energy $E_{p}$ strikes on a semiconductor material, it will be reflected, transmitted, or absorbed. Here we only consider the portion that is absorbed. Some of absorbed light energy inelastically interact with electrons causing them transit from energy states in conduction band to those of valence band emitting light with energy of $\hbar \omega$. We focus on the interaction of the electrons and photons resulting in light emission when electrons make the interband downward transition $\Gamma_{\downarrow}$. When an electromagnetic wave is propagating through a semiconductor, only interband transition in which the initial and final states of the electron belong to different bands is allowed according to 
both the energy and momentum conservation rules. It is a vertical transition in $k$-space as the optical momentum is much smaller than electron momentum causing no horizontal shift in the $k$-space. It is assumed the electron spin is conserved during the photon/electron interaction process. In 2D materials such as monolayer $\mathrm{MoS}_{2}$, for the event of excited electrons making their transition from a higher state $i$ in conduction band to a lower energy state $j$ in valence band, releasing new photons with identical optical properties (i.e. phase, frequency, polarization, and direction) as the incident wave, we shall use stimulated emission theory to calculate the transition rate. The emission rate for photons of energy $\hbar \omega$ is based on Fermi's golden rule [16], which is

$$
\Gamma_{i-j}=\frac{2 \pi}{\hbar}\left|W_{i j}\right|^{2} D_{3}^{o p}(\hbar \omega) B(\hbar \omega) f_{c}\left(1-f_{v}\right)
$$

where $\hbar$ is the reduced Planck's constant, $\left|W_{i j}\right|^{2}$ is the squared matrix element coupling the initial and the final states. $\left|W_{i j}\right|^{2}$ is determined by the electron wavevector $\mathrm{k}$ and the polarization direction of the electromagnetic wave. In most of III-V and II-IV semiconductors, the average value of the momentum matrix element can be treated as a constant. $D_{3}^{o p}(\hbar \omega)=\frac{(\hbar \omega)^{2} n_{r}^{2}}{\pi^{2} \hbar^{3} c^{3}}$ is the density of photon states, and $B(\hbar \omega)=\frac{1}{e^{\left(\hbar \omega / k_{B} T\right)}-1}$ is the Bose-Einstein occupation factor for photons. $n_{r}$ denotes the refractive index of the material, and $c$ represents the speed of light in air. $f_{c}$ and $f_{v}$ are Fermi-Dirac distribution functions of electron states in the conduction band and valence band respectively $\left(\frac{1}{e^{(E-\mu) / k_{B} T}+1}\right)$. “ $f_{c}\left(1-f_{v}\right)$ " essentially means that the transition rate depends on the presence of charge carriers in the conduction band, and the numbers of vacant sites in the valence band facilitating the transition to take place. The total emission $R_{s t}$ for specific photon energy is the sum of all energy levels separated by this photon energy, that is

$$
R_{S t}=\sum_{k} \Gamma_{i-j} \delta\left(E_{i j}-\hbar \omega\right)
$$

Delta function sets the constraint for the energy conservation. In equation (2), the integral of the Delta function with respect to $k, \int \frac{d^{3} k}{(2 \pi)^{3}} \delta\left(E_{i j}-\hbar \omega\right)$, is essentially the density of states $D_{3}(E)$ in three-dimension for the bulk materials. Density of states (DOS) is defined as the number of states in a conductor per unit energy. The DOS for bulk materials with no confinement is written as

$$
\begin{aligned}
& D_{3}(\hbar \omega)=\frac{1}{2 \pi^{2}}\left(\frac{2 m_{r}}{\hbar^{2}}\right)^{3 / 2}\left(\hbar \omega-E_{g}\right)^{1 / 2} \\
& \hbar \omega \geq E_{g}
\end{aligned}
$$

where the reduced effective mass $\frac{1}{m_{r}}=\frac{1}{m_{e}^{*}}+\frac{1}{m_{h}^{*}}$, $m^{*} e$ and $m_{h}^{*}$ are effective electron and hole mass respectively. $E_{g}=E_{c}-E_{v}$. For $2 \mathrm{D}$ materials such as monolayer $\mathrm{MoS}_{2}$, the DOS is a constant expressed as

$$
D_{2}(\hbar \omega)=\frac{m_{r}}{2 \pi \hbar^{2}}
$$$$
\hbar \omega \geq E_{g}
$$

Integrating the equation (2), and combining with equation (3) leads to the total emission rate for the bulk material

$$
\begin{aligned}
& R_{S t}=\frac{2 \pi}{\hbar} \frac{(\hbar \omega)^{2} n_{r}^{3}}{\pi^{2} \hbar^{3} c^{3}}\left|W_{i j}\right|^{2} B(\hbar \omega) \times \\
& \times \frac{1}{2 \pi^{2}}\left(\frac{2 m_{r}}{\hbar^{2}}\right)^{3 / 2}\left(\hbar \omega-E_{g}\right)^{1 / 2} f_{c}\left(1-f_{v}\right)
\end{aligned}
$$

For monolayer $\mathrm{MoS}_{2}$, electrons are likely confined in two dimensions, akin to the quantum well. Therefore stimulated emission $R_{s t-2 d}$ can be obtained from the equations (2) and (4), which is 
$R_{S t-2 D}=\frac{2 \pi}{\hbar} \frac{(\hbar \omega)^{2} n_{r}^{3}}{\pi^{2} \hbar^{3} c^{3}}\left|W_{i j}\right|^{2} \times$

$\times B(\hbar \omega) \frac{m_{r}}{2 \pi \hbar^{2}} f_{c}\left(1-f_{v}\right)$

Re-arrange equations (5) and (6), let $A=\left|W_{i j}\right|^{2}$, they become

$$
\begin{aligned}
& R_{S t}=\frac{2 \sqrt{2} m_{r}^{3 / 2} n_{r}^{3} A}{\pi^{3} \hbar^{7} c^{3}}(\hbar \omega)^{2} B(\hbar \omega) \times \\
& \times\left(\hbar \omega-E_{g}\right)^{1 / 2} f_{c}\left(1-f_{v}\right) \\
& R_{S t-2 d}=\frac{m_{r} n_{r}^{3} A}{\pi \hbar^{6} c^{3}}(\hbar \omega)^{2} B(\hbar \omega) f_{c}\left(1-f_{v}\right)
\end{aligned}
$$

Finally the scaled stimulated emissions for both scenarios $\left(R_{S t}^{\prime}=\frac{R_{S t} \pi^{3} \hbar^{7} c^{3}}{2 \sqrt{2} m_{r}^{3 / 2} n_{r}^{3} A}, R_{S t-2 d}^{\prime}=\frac{R_{S t-2 d} \pi \hbar^{6} c^{3}}{m_{r} n_{r}{ }^{3} A}\right)$ are given by

$$
\begin{aligned}
& R_{S t}^{\prime}=(\hbar \omega)^{2} B(\hbar \omega)\left(\hbar \omega-E_{g}\right)^{1 / 2} f_{c}\left(1-f_{v}\right) \\
& R_{S t-2 d}^{\prime}=(\hbar \omega)^{2} B(\hbar \omega) f_{c}\left(1-f_{v}\right)
\end{aligned}
$$

Clearly the emission rate due to photon-electron interaction is proportional to squared incident energy, Bose-Einstein distributions of photons, square root of the energy difference between incident wave and the band gap of the material, and the electrons occupation distributions in both bands for the bulk $\mathrm{MoS}_{2}$ material. For monolayer $\mathrm{MoS}_{2}$, as the 2D DOS can be treated as a constant, the total emission rate no longer depends on the bandgap $E_{g}$. Further quantitative study based on aforementioned theory is described in the next section.

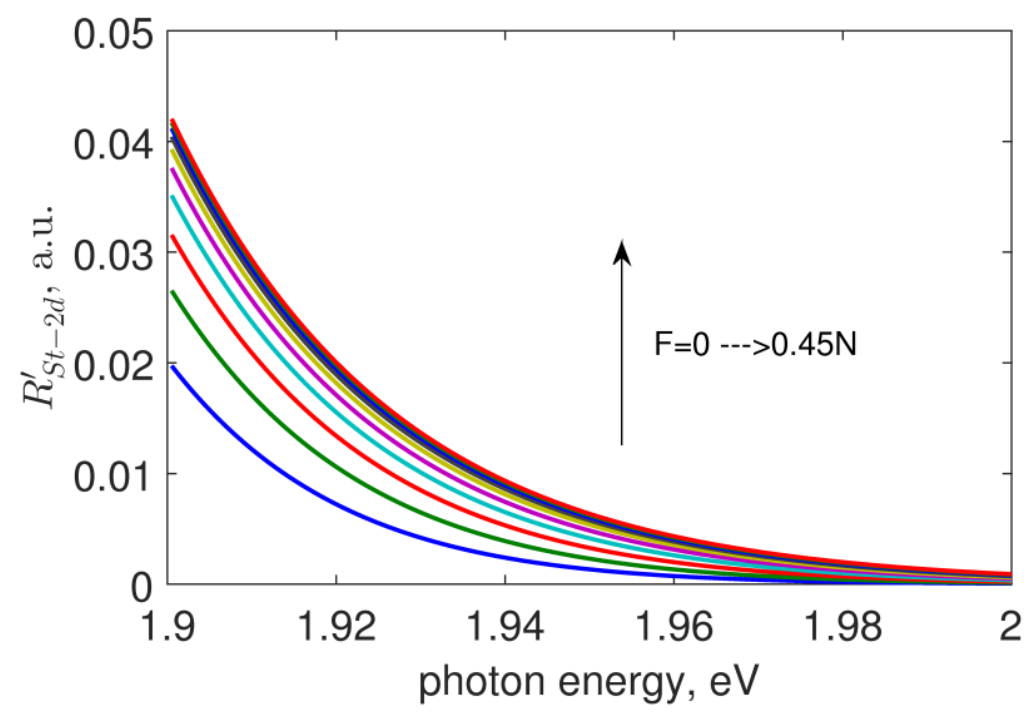

Figure 2, Calculation results of the relation between the emission and the incident photon energy $\hbar \omega$ under an increasing external force $F$ for the $2 \mathrm{D} \mathrm{MoS}_{2}$.

\section{Results and Analysis}

According to the equation (8), we calculate the stimulated emissions numerically. Dispersion in the refractive index is not considered in this simulation. The chemical potential for a number of electrons and holes per unit volume at certain temperatures is required to calculate in order to obtain $f_{c}$ and $1-f_{v}$. The chemical potential is determined by carrier density and temperature. Taking the spin degeneracy of 2 into consideration, the relation between the chemical potential and carrier density at a fixed temperature for a three-dimensional electron gas (bulk material) is

$$
\begin{aligned}
& n=\int_{0}^{\infty} D_{3}(E) 2 f(E) d E=\frac{1}{2 \pi^{2}}\left(\frac{2 m}{\hbar^{2}}\right)^{3 / 2} \times \\
& \times \int_{E_{\min }=0}^{E_{\max }=\infty} E^{1 / 2} \frac{1}{e^{(E-\mu) / k_{B} T}+1} d E
\end{aligned}
$$

To obtain the chemical potential $\mu$ numerically, the procedure in reference [14] is followed. The maximum chemical potential is given by the Fermi 
energy $\quad \mu_{\max }=E_{F}=\frac{\hbar^{2} k_{F}^{2}}{2 m} \quad k_{F}=\left(3 \pi^{2} n_{c}\right)^{1 / 3}, n_{c}$ is the carrier density. The minimum chemical potential at high temperature limit is given by $\mu_{\min }=k_{B} T \ln \left(\frac{n}{2}\left(\frac{2 \pi \hbar^{2}}{m k_{B} T}\right)^{3 / 2}\right)$. Same approach can be adopted for the 2D materials, in which case the 2D DOS is used in the equation (9), which becomes

$n=\int_{0}^{\infty} D_{2}(E) 2 f(E) d E=\frac{m}{\pi \hbar^{2}} \int_{E_{\min }=0}^{E_{\max }=\infty} \frac{1}{e^{(E-\mu) / k_{B} T}+1} d E$

The chemical potential can be derived analytically from the above integral for quasi-Fermi levels of electrons and holes (the material is assumed undoped). They are expressed as

$\mu_{e}=-k_{B} T \ln \left[\frac{1-\exp \left(\left(\frac{n \pi \hbar^{2}}{m}-E_{m}\right) /\left(k_{B} T\right)\right)}{\exp \left(\frac{n \pi \hbar^{2}}{m k_{B} T}\right)-1}\right]$
$\mu_{h}=-k_{B} T \ln \left[\frac{1-\exp \left(\frac{n \pi \hbar^{2}}{m k_{B} T}\right)}{\exp \left(\left(\frac{n \pi \hbar^{2}}{m}-E_{m}\right) /\left(k_{B} T\right)-1\right)}\right]$

The calculated quasi-Fermi levels for electrons and holes are then used in equation (8) to calculate the stimulated emission rate. In the calculation, effective electron and heavy hole mass are taken as $0.37 m_{0}$ and $0.44 m_{0}$ respectively, where $m_{0}$ is the electron mass [17]. Temperature is set to $300 \mathrm{~K}$. The bulk $\mathrm{MoS}_{2}$ crystal usually has an indirect-gap of $1.29 \mathrm{eV}$, and a direct band gap of $1.90 \mathrm{eV}$ for the monolayer $\mathrm{MoS}_{2}$ [2]. It is noted that in principle, the photon energy cannot assist the non-vertical interband electron transition. For the piezoelectric enhanced emission, we shall consider the single layer material. The refractive index of the monolayer $\mathrm{MoS}_{2}$ was measured previously, and the real part of the refractive index is around 4 at the wavelength range of $450 \mathrm{~nm}$ to 750 $\mathrm{nm}$ [18].

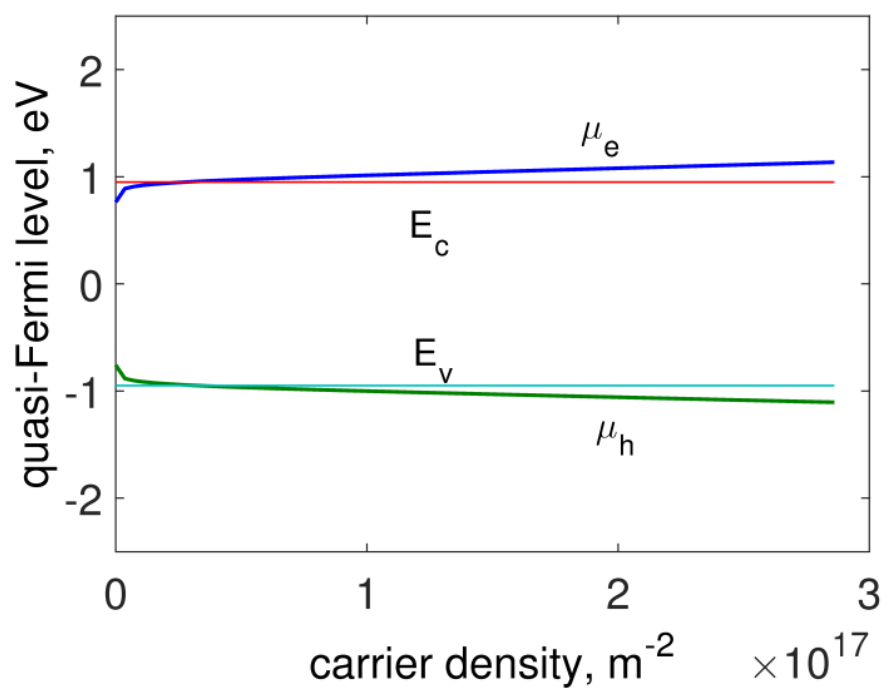

Figure 3, Simulated quasi-Fermi levels for electrons and holes when varying carrier density. As carrier density increases, $\mu_{e}$ and $\mu_{h}$ gradually entre to conduction and valence band, eventually leading to saturation of the total emission. Here we set the middle of the bandgap as 0.

The carrier density for the undoped monolayer $\mathrm{MoS}_{2}$ was reported to be $\sim 10^{13} \mathrm{~cm}^{-2}$ [19]. Piezoelectric constant $e_{11}$ was reported as $0.5 \mathrm{C} / \mathrm{m}^{2}$ or $2.9 \times 10^{-10}$ $\mathrm{C} / \mathrm{m}$ normalized by the thickness [7] [20]. The corresponding piezoelectric strain tensor $\left(d_{11}\right)$ of the material is predicted from the following relation [21] $d_{11}=e_{11} /\left(C_{11}-C_{12}\right)$, where $C_{11}$ and $C_{12}$ are planar elastic stiffness coefficients, which are $132.7 \mathrm{~N} / \mathrm{m}$ and 33.0
$\mathrm{N} / \mathrm{m}$ based on the relaxed ion calculation, $d_{11}$ is then calculated to be $2.9 \times 10^{-12} \mathrm{C} / \mathrm{N}$. The size of the monolayer $\mathrm{MoS}_{2}$ is designed as $5 \mu \mathrm{m}$ width and 10 $\mu \mathrm{m}$ length, with thickness of a single layer atom. Constituent piezoelectric equations describing relationships between electrical and mechanical behaviour of the structure are 
$S=s^{E} T+d E$

$D=d T+\varepsilon^{T} E$

where $S, T, E, D$ are strain, stress, electrical filed strength, and dielectric displacement respectively. $s, d$ $\varepsilon$ are mechanical compliance, piezoelectric constant, and dielectric constant, where the superscripts to the symbols denote the quantity kept constant under boundary conditions. For this case, as the mechanical-to-electrical conversion and the optical behaviour will be investigated, the electrical circuit connecting two ends of the $\mathrm{MoS}_{2}$ ribbon is open, assuming there is no external electrical field the equation (13) is simplified to $D=d T$. The stretching force $F$ is applied on both ends of the ribbon along the $x$-axis (Figure 1). The strain induced piezoelectric charges will be accumulated on both sides of the ribbon, the induced charge $Q=d_{11} F, d_{11}$ has been specified in the previous section. The force is set varying from 0 to $0.45 \mathrm{~N}$, the increment of carrier concentration is calculated from the initial set value $0.5 \times 10^{17} \mathrm{~m}^{-2}$ up to $2.1 \times 10^{17} \mathrm{~m}^{-2}$. The total stimulated emission vs. incident photon energy $\hbar \omega$ is calculated and shown in Figure 2. It is seen that the emission intensity increases with the increasing external force (carrier density). For a fixed external force, the emission is at maximum when the photon energy equals to the direct bandgap of the material. The emission reaches to a saturation point for large carrier densities. Quasi Fermi levels for electrons and holes have been calculated and results are shown in Figure 3, where we set 0 to the middle of the bandgap for demonstration purpose. While conduction band and valence band edges are set to 0 in the calculation. It is seen that for a small carrier density value, $\mu_{e}$ and $\mu_{h}$ are within the bandgap. As the external force increases, the piezoelectric induced charges cause them deviate from each other, and enter to conduction and valence bands respectively. Figure 2 shows the emission intensity approaches to a saturation point at a large carrier density, it is imperative to unveil the detailed relationship between photons energy, carrier density, and emission intensity. Figure 4 display the results showing that emission increases rapidly in the region of 0 to $1.5 \times 10^{17} \mathrm{~m}^{-2}$, and the emission remains unchanged after $2 \times 10^{17} \mathrm{~m}^{-2}$. It is noted that this is the simulation based on static response of the piezoelectric, and screening effect on piezoelectric potential [22] is not considered, which may reduce the luminescence as time evolves. In this work, only single layer $\mathrm{MoS}_{2}$ is considered because it exhibits pure direct bandgap. The thickness consideration in [5] is facilitated by multiple-layers, which is not taken into consideration in this paper. We neglect the edge effect in this work. The edge effect will be considered in our further device model which include electrodes.

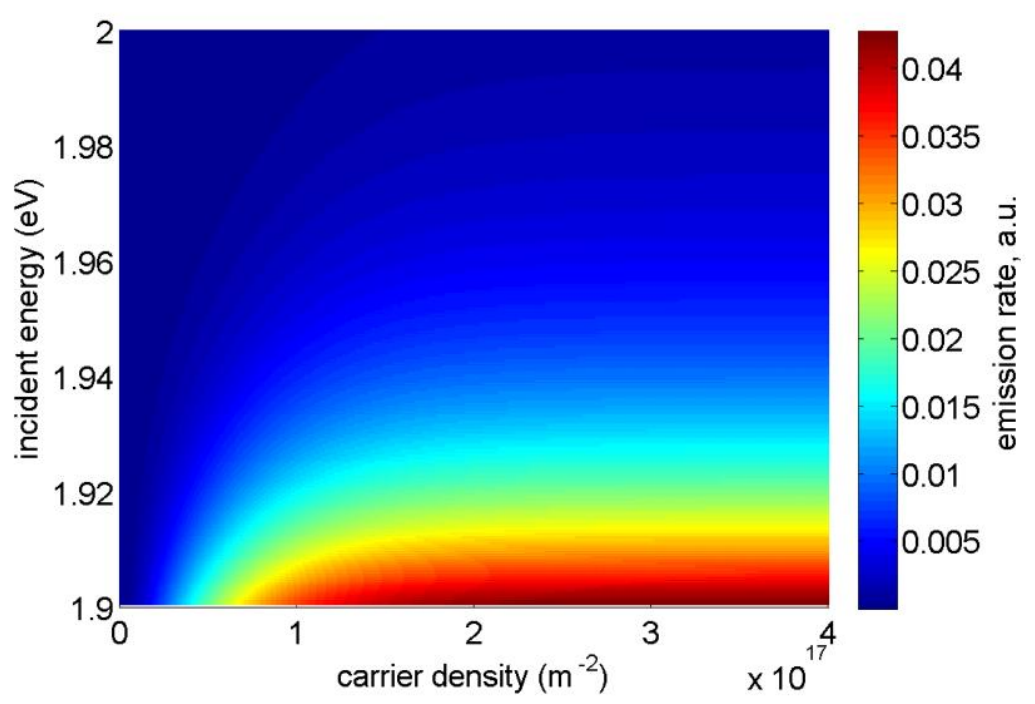

Figure 4, Total emission rate vs. incident energy and carrier density. 
In [23], the 2D material is used together with a drain and a source to form the transistor type device, where the drain voltage is constantly applied. But our simulation is one of simplified models where the 2D material is purely connected to two mechanical pads that can be dielectric, and no drain voltage is applied. When the transistor type device is structured, consideration of charge transportation (including the screening effect) through the material dominates, whereas we only simulate the static state, i.e. when the electrode is considered, the conduction band of the $\mathrm{MoS}_{2}$ will be pulled down/up on the metal-semiconductor interfaces, forming Schottky junctions. However the direct bandgap remains unchanged and the photon energy will not change. The emission intensity changes with the depletion region at the interfaces. Because the model does not involve electron transportation through the material and electrodes, the junction effect is neglected in this work. In fact the pads attaching to the $2 \mathrm{D} \mathrm{MoS}_{2}$ can be a dielectric material, as they are only used for conveniently applying mechanical forces. piezoelectric induced charges increase the carrier density, hence increasing the luminescence. When a static force is applied, piezoelectric charges are generated at the interface, and distribute in a nano scale. The width of piezoelectric charge distribution can be treated as uniform in $2 \mathrm{D}$ material as a simplified model, which is used in our previous $\mathrm{ZnO}$ nanowire transistor model [24]. The width of piezoelectric charge distribution has been calculated by first principle simulations in piezoelectric semiconductor nanowire, such as $\mathrm{ZnO}$ and GaN [25]. It is found that the width of piezoelectric charge distribution depended on not only different piezoelectric semiconductor materials, but also the metal electrodes of devices. The width of piezoelectric charge distribution will be calculated in our further work by the density function theory.

The applied strain induces piezoelectric charges, subsequently increases the carrier density in the material. The lattice deformation is not considered in the calculation, the qualitative prediction is that the crystal structure is being stretched by the mechanical force, hence inducing the change of the second harmonic generation (SHG) spectrum. Since the piezoelectric property of the monolayer $\mathrm{MoS}_{2}$ is due to lack of inversion symmetry, which also allows strong optical SHG [26] [27] [5] [28] [29]. As reported previously, for the parallel polarization component, the $\mathrm{SH}$ intensity from monolayer $\mathrm{MoS}_{2}$ exhibited a six-fold symmetric response as a function of azimuthal angle $\theta$ for rotation about its surface normal (Figure 5a), where $\theta=0$ implies the incident beam is parallel to the mirror plane (horizontal line) of the crystal. The $\mathrm{SH}$ intensity is governed by $I_{2 \omega}=I_{0} \cos ^{2}\left(3 \theta+\theta_{0}\right) \quad$ [27] [28] [26]. Figure 5b shows the $\mathrm{SH}$ intensity for the parallel polarization component. It is straightforward to envisage that when a mechanical force $F$ is stretching the monolayer $\mathrm{MoS}_{2}$ as shown in Figure 5c, the crystal structure is skewed, causing change of the $\mathrm{SH}$ angular spectrum, especially for the polarization angles $60^{\circ}, 120^{\circ}, 240^{\circ}$, and $300^{\circ}$. Equivalently, new crystal orientations causes the intensity function become $I_{2 \omega}^{\text {skewed }}=I_{0} \cos ^{2}\left(3 \theta \pm \theta_{\text {offset }}\right)$. Calculation for $\theta= \pm 25^{\circ}$ has been shown in the Figure 5c. From previous sections of this paper it is clearly to see that the mechanical force enables manipulation of the stimulated emission as well as SHG due to the non-centrosymmetric property of the monolayer $\mathrm{MoS}_{2}$.

\section{Conclusion}

We can conclude from the analysis that for the 2D monolayer $\mathrm{MoS}_{2}$, stimulated emission can be simulated from the theory of photon-solid interaction. The main difference between the bulk and 2D MoS 2 in terms of the theoretical mechanism is due to the different density of states. For 2D material, the emission is peaked at the bandgap value and decreases as the photon energy increases. This is due to the decreasing number of energy states above conduction band minimum and below the valence band maximum. As the single layer $\mathrm{MoS}_{2}$ demonstrates piezoelectric property, simulation has also been conducted to unveil the impact on emission by the piezoelectric effect. It is shown that the emission is enhanced by the increasing piezoelectric 
charges in the low carrier density region. At high carrier density region, the emission eventually saturates. SHG from the single layer $\mathrm{MoS}_{2}$ also changes subjecting to the external mechanical force. This study paves the way for further exploitation of the $2 \mathrm{D} \mathrm{MoS}_{2}$ in light emitting devices.

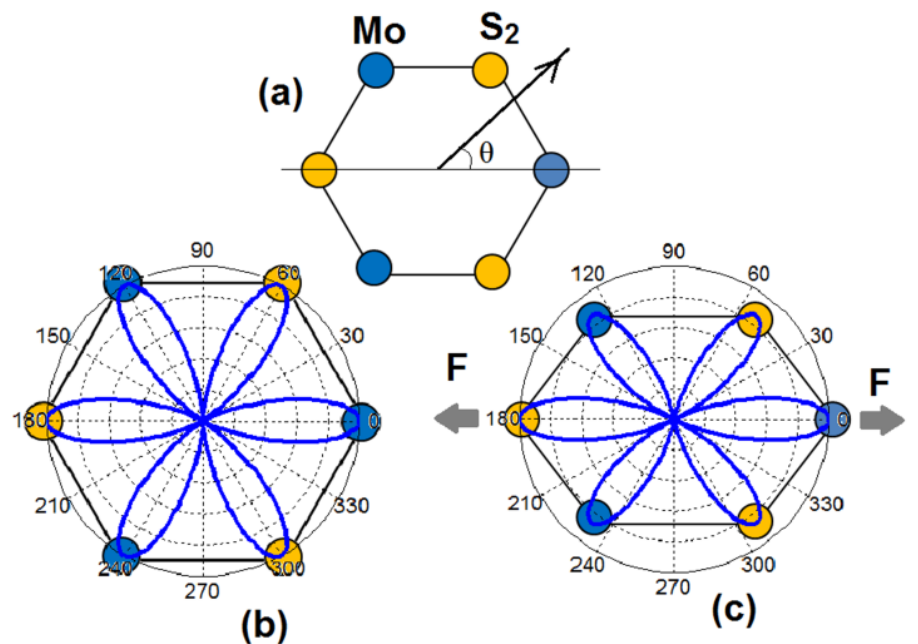

Figure 5, Polar plot of the SH intensity from monolayer $\mathrm{MoS}_{2}$ as a function of $\theta$. (a) schematic of the crystal structure. (b) Simulated polar plot of SH intensity without mechanical force. (c) Simulated polar plot of SH intensity with a stretching force $F$ in the horizontal direction. The graph shows polarization angle has been changed by the distortion of the crystal structure.

\section{Acknowledgements}

We would like to thank Swansea University, UK and University of Electronic Science and Technology of China for support.

\section{References}

[1] Radisavljevic, B.;Radenovic, A.;Brivio, J.;Giacometti, V.; Kis, A. Single-layer $\mathrm{MoS}_{2}$ transistors. Nature Nanotechnology 2011, 6, 147-150.

[2] Mak, K. F.;Lee, C.;Hone, J.;Shan, J.; Heinz, T. F. Atomically Thin $\mathrm{MoS}_{2}$ : A New Direct-Gap Semiconductor. Physical Review Letters 2010, 105, 136805.

[3] Wang, Q. H.;Kalantar-Zadeh, K.;Kis, A.;Coleman, J. N.; Strano, M. S. Electronics and optoelectronics of two-dimensional transition metal dichalcogenides.

Nature Nanotechnology 2012, 7, 699-712.

[4] Splendiani, A.;Sun, L.;Zhang, Y. B.;Li, T. S.;Kim,
J.;Chim, C. Y.;Galli, G.; Wang, F. Emerging Photoluminescence in Monolayer $\mathrm{MoS}_{2}$. Nano Letters 2010, 10, 1271-1275.

[5] Wu, W.;Wang, L.;Li, Y.;Zhang, F.;Lin, L.;Niu, S.;Chenet, D.;Zhang, X.;Hao, Y.;Heinz, T. F.;Hone, J.; Wang, Z. L. Piezoelectricity of single-atomic-layer $\mathrm{MoS}_{2}$ for energy conversion and piezotronics. Nature 2014, 514, 470-474.

[6] Chen, L. B.;Xue, F.;Li, X. H.;Huang, X.;Wang, L. F.;Kou, J. Z.; Wang, Z. L. Strain-Gated Field Effect Transistor of a $\mathrm{MoS}_{2} \mathrm{ZnO} 2 \mathrm{D}-1 \mathrm{D}$ Hybrid Structure. Acs Nano 2016, 10, 1546-1551.

[7] Zhu, H.;Wang, Y.;Xiao, J.;Liu, M.;Xiong, S.;Wong, Z. J.;Ye, Z.;Ye, Y.;Yin, X.; Zhang, X. Observation of piezoelectricity in free-standing monolayer $\mathrm{MoS}_{2}$. Nat Nano 2015, 10, 151-155.

[8] Splendiani, A.;Sun, L.;Zhang, Y.;Li, T.;Kim, J.;Chim, C.-Y.;Galli, G.; Wang, F. Emerging

Photoluminescence in Monolayer $\mathrm{MoS}_{2}$. Nano Letters 
2010, $10,1271-1275$.

[9] Wang, Z. L. From nanogenerators to piezotronics-A decade-long study of $\mathrm{ZnO}$ nanostructures. MRS Bulletin 2012, 37, 814-827.

[10] Wang, Z. L. Piezotronics and Piezo-Phototronics; Springer-Verlag: Berlin/Heidelberg, Germany, 2012.

[11] Pan, C.;Chen, M.;Yu, R.;Yang, Q.;Hu, Y.;Zhang, Y.; Wang, Z. L. Progress in

Piezo-Phototronic-Effect-Enhanced Light-Emitting Diodes and Pressure Imaging. Advanced Materials 2016, 28, 1535-1552.

[12] Wong, M.-C.;Chen, L.;Tsang, M.-K.;Zhang, Y.; Hao, J. Magnetic-Induced Luminescence from Flexible Composite Laminates by Coupling Magnetic Field to Piezophotonic Effect. Advanced Materials 2015, 27, 4488-4495.

[13] Bai, G.;Tsang, M.-K.; Hao, J. Tuning the Luminescence of Phosphors: Beyond Conventional Chemical Method. Advanced Optical Materials 2015, $3,431-462$.

[14] Zhang, Y.; Li, L. Piezophototronic effect enhanced luminescence of zinc oxide nanowires. Nano Energy 2016, 22, 533-538.

[15] Bai, G.;Yuan, S.;Zhao, Y.;Yang, Z.;Choi, S. Y.;Chai, Y.;Yu, S. F.;Lau, S. P.; Hao, J. 2D Layered Materials of Rare-Earth Er-Doped $\mathrm{MoS}_{2}$ with NIR-to-NIR Down- and Up-Conversion Photoluminescence. Advanced Materials 2016, 28, 7472-7477.

[16] Parker, M. A. Physics of Optoelectronics; CRC Press: Boca Raton, 2005.

[17] Peelaers, H.; Van de Walle, C. G. Effects of strain on band structure and effective masses in $\mathrm{MoS}_{2}$.

Physical Review B 2012, 86, 241401.

[18] Zhang, H.;Ma, Y.;Wan, Y.;Rong, X.;Xie, Z.;Wang, W.; Dai, L. Measuring the Refractive Index of Highly Crystalline Monolayer $\mathrm{MoS}_{2}$ with High Confidence. Scientific Reports 2015, 5, 8440.

[19] Mouri, S.;Miyauchi, Y.; Matsuda, K. Tunable Photoluminescence of Monolayer $\mathrm{MoS}_{2}$ via Chemical Doping. Nano Letters 2013, 13, 5944-5948.
[20] Zhou, Y.;Liu, W.;Huang, X.;Zhang, A.;Zhang, Y.; Wang, Z. L. Theoretical study on two-dimensional $\mathrm{MoS}_{2}$ piezoelectric nanogenerators. Nano Research 2016, 9, 800-807.

[21] Alyörük, M. M.;Aierken, Y.;Çakır, D.;Peeters, F. M.; Sevik, C. Promising Piezoelectric Performance of Single Layer Transition-Metal Dichalcogenides and Dioxides. The Journal of Physical Chemistry C $\mathbf{2 0 1 5}$ 119, 23231-23237.

[22] Baek, S. K.;Kwak, S. S.;Kim, J. S.;Kim, S. W.; Cho, H. K. Binary Oxide p-n Heterojunction Piezoelectric Nanogenerators with an Electrochemically Deposited High p-Type $\mathrm{Cu}_{2} \mathrm{O}$ Layer. ACS Applied Materials \& Interfaces 2016, 8, 22135-22141.

[23] Wu, W.;Wang, L.;Yu, R.;Liu, Y.;Wei, S.-H.;Hone, J.; Wang, Z. L. Piezophototronic Effect in Single-Atomic-Layer $\mathrm{MoS}_{2}$ for Strain-Gated Flexible Optoelectronics. Advanced Materials 2016, 28, 8463-8468.

[24] Zhang, Y.;Liu, Y.; Wang, Z. L. Fundamental Theory of Piezotronics. Advanced Materials 2011, 23, 3004-3013.

[25] Liu, W.;Zhang, A.;Zhang, Y.; Lin Wang, Z. First principle simulations of piezotronic transistors. Nano Energy 2015, 14, 355-363.

[26] Kumar, N.;Najmaei, S.;Cui, Q.;Ceballos, F.;Ajayan, P. M.;Lou, J.; Zhao, H. Second harmonic microscopy of monolayer $\mathrm{MoS}_{2}$. Physical Review B 2013, 87, 161403

[27] Malard, L. M.;Alencar, T. V.;Barboza, A. P. M.;Mak, K. F.; de Paula, A. M. Observation of intense second harmonic generation from $\mathrm{MoS}_{2}$ atomic crystals. Physical Review B 2013, 87, 201401.

[28] Li, Y.;Rao, Y.;Mak, K. F.;You, Y.;Wang, S.;Dean, C. R.; Heinz, T. F. Probing Symmetry Properties of Few-Layer $\mathrm{MoS}_{2}$ and h-BN by Optical Second-Harmonic Generation. Nano Letters 2013, 13, 3329-3333

[29] Jiang, T.;Liu, H.;Huang, D.;Zhang, S.;Li, Y.;Gong, X.;Shen, Y.-R.;Liu, W.-T.; Wu, S. Valley and band 
structure engineering of folded $\mathrm{MoS}_{2}$ bilayers. Nat

Nano 2014, 9, 825-829. 